\title{
Adult Brain Medulloblastoma
}

National Cancer Institute

\section{Source}

National Cancer Institute. Adult Brain Medulloblastoma. NCI Thesaurus. Code C9373.

A medulloblastoma arising from the brain, occurring in adults. 